\title{
Using FRAP as a Technique to Quantitate Local Reactive Oxygen Species (ROS) Production
}

Josh Hughes ${ }^{1}$, David Chisholm ${ }^{2}$, Carrie Ambler ${ }^{2}$, Andrew Whiting ${ }^{2}$ and John Girkin ${ }^{1}$

${ }^{1}$ Durham University, Durham, England, United Kingdom, ${ }^{2}$ LightOx Ltd, Billingham, England, United Kingdom

\section{Introduction}

FRAP (Fluorescence Recovery After Photobleaching) is a technique that measures the recovery of fluorescence intensity in a specified region of the cell following photobleaching. This technique, first developed in the 1970s, is primarily utilised to probe diffusion, motility and dynamics of proteins within the cell membrane.

Reactive oxygen species (ROS) are highly reactive molecules that oxidise macromolecules, causing oxidative stress, which in high quantities can trigger apoptosis. Photodynamic Therapy (PDT) employs this principle in a two-step process to kill biological cells. A photosensitiser, a light activated molecule, and a light source are needed in conjunction to produce ROS, which in turn will trigger apoptosis. By using FRAP we can quantify ROS production by measuring the fluorescence intensity of CellROX, a ROS reporter, following photo-bleaching of LightOx58, a photosensitiser.

\section{Methods/Materials}

HaCaT keratinocytes cell lines were plated on $22 \times 22 \mathrm{~mm}$ cover slips and incubated with; LightOx58, LightOx58 and CellROX, CellROX. LightOx58 was incubated for 1.5 hours at a concentration of 1uM, and CellROX was incubated for 30mins at a concentration of 5uM. Cover slips were then washed in PBS and wet mounted on microscope slides. A Leica Sp5 was used for the imaging using the FRAP wizard feature. $405 \mathrm{~nm}$ laser light was used to irradiate LightOx58 at 100\% laser power, 633nm laser was used to image CellROX at $10 \%$ laser power. Each region of interest was a 4 um diameter circle, focused on the mitochondria of the cells.

\section{Results and Discussion}

Historically, FRAP was first used to test the motility and dynamics of a compound by measuring the fluorescence recovery. Here, FRAP is used to quantify the two-step production of ROS.

Data were normalised so that the fluorescence prior to bleaching has a fluorescent intensity value of 1 . Diffusion constants and immobile fractions were then derived from the curvature and long-term value of fluorescence intensity, . Importantly, in the absence of new fluorescence production, cannot exceed 1. This premise is diagrammed in the all the controls, in which one fluorophore was bleached by one wavelength of light. Furthermore, these controls consistently had mobile fraction values (the fraction of fluorophore free to diffuse) lower than one.

However, by using a two-step production of ROS, via excitation of LightOx58, we were able to produce new fluorescence of CellROX. These ROS molecules bind to inactive reduced CellROX molecules, oxidising them into their active fluorescent state. As there are more active fluorescing CellROX molecules after the bleaching phase than before, the fluorescence intensity can rise above the threshold of 1 . Therefore, having a recovery curve that exceeds unity indicates ROS production. The resultant fluorescent intensity curves exceeded one, with a mobile fraction value of 2.4+/- 0.4 which indicates that there is ROS production. 


\section{Conclusions}

Here we have demonstrated a novel use of FRAP which was sufficient to demonstrate localised ROS production following a two-way activation process of a photosensitiser in a biological cell, using CellROX a ROS reporter.

\section{References}

Kang, M., Day, C.A., Kenworthy, A.K. and DiBenedetto, E., 2012. Simplified equation to extract diffusion coefficients from confocal FRAP data. Traffic, 13(12), pp.1589-1600. 